\title{
La politique au-delà des frontières : la sociologie politique de l'émigration
}

Politics beyond Borders: The Political Sociology of Emigration

Política más allá de las fronteras: la sociología política de la emigración

\section{Roger Waldinger}

\section{(2) OpenEdition}

Édition électronique

URL : https://journals.openedition.org/remi/8385

DOI : 10.4000/remi.8385

ISSN : $1777-5418$

Éditeur

Université de Poitiers

Édition imprimée

Date de publication : 1 décembre 2016

Pagination : 319-334

ISBN : 979-10-90426-29-0

ISSN : 0765-0752

\section{Référence électronique}

Roger Waldinger, «La politique au-delà des frontières : la sociologie politique de l'émigration », Revue européenne des migrations internationales [En ligne], vol. 32 - n³ et 4 | 2016, mis en ligne le 01 décembre 2018, consulté le 14 avril 2022. URL : http://journals.openedition.org/remi/8385 ; DOI : https://doi.org/10.4000/remi.8385 


\section{La politique au-delà des frontières : la sociologie politique de l'émigration}

\section{Roger Waldinger ${ }^{1}$}

Les mouvements de population à travers les frontières nationales représentent un défi à l'isomorphie des États, sociétés et peuples que soulignent tout à la fois les sciences sociales et le sens commun. C'est pourquoi la migration internationale est un phénomène proprement politique bien ce soit un aspect largement négligé par la sociologie des migrations. La majorité des chercheurs en sociologie politique des migrations s'intéressent aux sociétés d'accueil et traitent des questions - liées mais distinctes - de la politique migratoire et de la politique des migrants. La première porte sur deux aspects : les politiques concernant les passages de la frontière qu'il s'agisse de la frontière - extérieure - du territoire, ou de la frontière - intérieure - de la citoyenneté ; les droits dont jouissent les étrangers résidant sur le territoire d'un autre peuple. Quant à la littérature sur la politique des migrants, elle concerne les moyens par lesquels les étrangers s'engagent dans la politique, en apprennent les règles du jeu et, lorsqu'ils acquièrent la citoyenneté, entrent dans le système politique.

En revanche, pour une autre sociologie le centre d'intérêt réside plutôt dans les relations entre les États d'origine et leurs émigrés (Green et Weil, 2006). La recherche porte alors sur la politique des émigrés, la manière dont ceux-ci s'engagent à l'égard de l'État d'origine : soit pour détruire un État multiethnique afin d'établir un nouvel État avec son propre peuple; soit pour renverser un régime politique auquel ils s'opposent ; soit pour agir comme un lobby ethnique, préconisant les intérêts de l'État émetteur chez l'État récepteur ; soit encore pour changer les lois électorales ou de citoyenneté, afin de pouvoir voter de l'extérieur et accéder à la double citoyenneté (Dufoix, 2002 ; OstergaardNielsen, 2003 ; Shain, 1989). Plus récemment, s'est développée une littérature qui s'oriente plutôt vers les politiques que développent les États émetteurs envers leurs expatriés pour résoudre les problèmes que rencontrent les citoyens qui résident à l'étranger ou pour rétablir ou renforcer leurs liens à la société d'origine (Dufoix et al., 2010 ; Gamlen, 2008 ; Iskander, 2010). C'est sur cette littérature que se fonde cet article dans lequel j'essaie de développer une perspective analytique sur la sociologie politique de l'émigration ${ }^{2}$. Ce texte souligne la

\footnotetext{
1 Sociologue, Professeur, Département de sociologie, 264 Haines Hall, Université de Californie, Los Angeles 90095, Etats-Unis ; waldinge@soc.ucla.edu

2 Çet article a été préparé lors d'un séjour que j'ai effectué en tant que Directeur d'Études Associé du 15 mai au 15 juin 2012 à Paris, dans le cadre du programme "TIC-Migrations " de la Fondation de la Maison des Sciences de l'Homme.
} 
dualité logée au cœur du phénomène migratoire : les immigrants sont aussi des émigrants ; les étrangers au sens juridique (aliens) sont aussi des citoyens et les étrangers, au sens social (foreigners), sont aussi des nationaux; les exclus de la société de réception sont aussi des membres des sociétés d'origine. De la société émettrice, mais n'étant plus dans cette société, les migrants sont des membres dont les connexions transfrontalières et les besoins d'adaptation poussent l'État émetteur à agir au-delà des frontières ; cependant, le fait de résider à l'étranger et les changements de comportements et de valeurs affaiblissent leurs propres revendications d'appartenance. Puisque les émigrés se trouvent dans la société de réception, mais ne sont pas de cette société, ils en tirent des avantages, mais subissent également des inconvénients. L'accès aux territoires des pays riches fournit des ressources politiques et économiques qui leur permettent d'exercer de l'influence chez eux, influence dont ils ne bénéficiaient pas avant de partir pour l'étranger. En revanche, en tant que personnes résidant dans un pays qui leur est socialement étranger ils sont vulnérables; en tant que personnes résidant dans un pays sans être citoyens, leurs droits sont limités. Ces diverses situations déclenchent des interventions des États émetteurs qui cherchent à protéger et à influencer leurs nationaux résidant à l'étranger, mais également à répondre aux revendications de ces citoyens à l'extérieur du pays et à les canaliser afin de mieux les récupérer. Cependant, l'extension au territoire d'un pays étranger limite l'exercice du pouvoir ne permettant que l'exercice d'une influence. De plus, des interventions même limitées courent le risque d'enflammer les passions des nationaux qui déjà inquiets de la présence d'une population étrangère pourraient percevoir le resserrement des liens entre les étrangers et leur pays d'origine comme une menace encore plus grande.

Cet article se divise en trois parties, chacune cernant une des dimensions de la relation des émigrants avec l'État-nation dont ils sont les ressortissants. Tout d'abord, la dimension légale de la citoyenneté, puis la dimension identitaire liée à la qualité d'être membre de cet État et enfin la dimension de la participation politique. Mais avant d'entrer dans cette discussion, je voudrais présenter des éléments de la littérature sur le transnationalisme par rapport à laquelle je me positionne.

\section{Transnationalisme}

Cet article fait partie d'une recherche pour repenser les phénomènes du transnationalisme (Waldinger et Fitzgerald, 2004 ; Waldinger, 2006 ; Waldinger, 2011 ; Waldinger, 2015). La littérature à leur sujet est vaste et ne cesse de s'étendre et cela même au-delà du constat central selon lequel les connexions transétatiques se développent partout où ont lieu les migrations internationales (les théoriciens du transnationalisme s'accordent sur ce point). Néanmoins, il me semble que I'on peut identifier quelques thèmes qui réapparaissent parmi les recherches qui défendent ce point de vue.

Concept presque inédit il y a vingt ans, et puisant son origine dans un secteur plutôt marginal d'une discipline restée à la périphérie de la recherche sur l'immigration américaine, le transnationalisme est rapidement devenu non seulement l'objet d'une intense recherche en sciences sociales, mais aussi une perspective distincte défendant l'importance, la persistance et la portée du 
champ social réunissant le " ici " des migrants avec un " là-bas " des parents, amis, communautés et mêmes nations d'origine (Basch et al., 1994 ; Levitt et Jaworsky, 2007 ; Saunier, 2009). Selon ce point de vue, les migrants ne sont plus contraints de rompre les liens avec tout ce qu'ils ont laissé dans leurs communautés de naissance. Au contraire, les changements technologiques facilitent les liens rattachant le " ici " et le "là-bas " car une réception plus bienveillante dans les pays d'arrivée encourage les migrants à maintenir leurs engagements avec les proches résidant encore dans les pays d'origine et que les pays d'émigration mettent en place des politiques dont l'objet est de maintenir la loyauté des " transmigrants " et d'influencer sur leurs démarches. Pour cette raison, l'époque contemporaine est caractérisée par l'implantation de " communautés transnationales ", donnant aux immigrés la possibilité inédite de vivre en deux endroits en même temps. Au lieu de l'assimilation subie par les migrants d'antan, ceux d'aujourd'hui connaissent un " processus d'incorporation simultanée dans les États d'origine et de peuplement".

Les théoriciens du transnationalisme ont souligné le foisonnement des liens, sociaux, économiques ou culturels rattachant les pays d'immigration aux pays d'origine. En traitant des aspects politiques, ils ont largement focalisé leur attention sur les " nouvelles formes d'interaction des États d'origine dans la politique du pays de réception et les formes d'intervention systématique des immigrants dans le pays d'origine " (Itzigsohn, 2000 : 1127).

Une formulation très courante met en relief les contrastes entre les activités des migrants et celles des États émetteurs. Pour les premiers, il s'agit d'un transnationalisme " par le bas " et pour les seconds par contraste d'un transnationalisme " par le haut " (Smith et Guarnizo, 1998). "Par le bas ", les émigrants cherchent à s'engager auprès des communautés politiques dans le pays d'origine en tentant de les influencer ou en visant une institutionnalisation de leur rôle dans la politique de l'État d'origine ou encore en essayant de changer les conditions dans leurs lieux d'origine spécifiques. "Par le haut ", les États émetteurs se rendent compte du fait que la transplantation d'une partie de leurs populations dans des pays riches est un bien que seul le hasard procure, pourvu que les expatriés ne coupent pas les liens les rattachant aux pays d'origine. Par conséquent, ils s'occupent des émigrés en leur fournissant des services, en essayant de les aider à résoudre leurs problèmes d'adaptation, en intervenant, là où ils le peuvent auprès des autorités et des politiciens, en faisant en somme tout ce qu'ils peuvent pour garantir un flot continu de remises et convertir les migrants en lobbyistes ethniques ayant la capacité de promouvoir les buts proposés par les gouvernements des sociétés d'origine.

Cependant, l'origine de ces interactions ne se trouve pas dans le champ social transnational mis en place par les migrations mais plutôt dans les frontières politiques qui coupent et divisent ce champ social. Paradoxalement, les activités entreprises par les migrants et qui dépassent le territoire sont le résultat des limites politiques de l'environnement récepteur. La richesse reste cantonnée à l'intérieur des États riches du Nord, ce qui ne sert pas seulement à produire les migrations, mais aussi à transformer celles-ci en un instrument utilisé afin de transférer les ressources d'un lieu favorisé à un autre plus pauvre. Bien que la traversée des frontières soit devenue de plus en plus difficile, ces mêmes frontières sont source de protection, soustrayant les immigrants à leur État 
d'origine et leur fournissant des ressources non seulement économiques, mais aussi politiques qui n'étaient pas disponibles avant de quitter la terre natale. Le matériel et le politique se rejoignent régulièrement : grâce à la richesse du pays de réception, ceux qui y ont du succès avancent modestement en tirant des avantages dans leurs rencontres avec les acteurs qui sont restés sur place. De plus, un système politique plus ouvert fournit des alliés, des compétences et des idées qui renforce l'influence des migrants quand ils s'engagent à l'égard des autorités dans le lieu d'origine. Par conséquent, au lieu d'être des communautés suspendues entre deux pays, pour citer Portes et Rumbaut (2006), certaines des populations immigrantes constituent des communautés contestées, autour desquelles les États d'origine et une variété de migrants sont en compétition pour capter les ressources et la loyauté des migrants.

Chez les théoriciens du transnationalisme, l'un des débats porte sur la question des interactions entre émigrants et États émetteurs. Pour certains, l'extension de l'État d'origine sur le sol de l'État récepteur représente une redéfinition de l'État. Selon ce point de vue, les États d'origine mettent en place une stratégie nouvelle consistant en ce que l'on dénomme le développement "d'un État-nation déterritorialisé ". La poursuite de cette stratégie repose sur l'idée que l'État-nation s'étend au-delà de ses limites géographiques. En comparaison avec les diasporas d'antan composées de peuples dispersés partout dans le monde sans accès à un État qui pourrait être le leur, la situation rencontrée par les migrants indique qu' " il $\mathrm{n}$ y a plus de diaspora, parce que où va son peuple, I'État y va aussi "(Basch et al., 1994 : 269). Malheureusement, cet argument réunit deux concepts, celui d'État et celui de nation, que la sociologie tend plutôt à différencier, le premier se référant aux unités territoriales, le second aux collectivités sociales. Par définition, les migrations internationales impliquent des connexions qui traversent les unités territoriales du monde mais la connectivité et la collectivité sociale sont pratiquement et analytiquement distinctes l'une de I'autre.

Les théoriciens du transnationalisme soulignent l'importance de l'incorporation politique ici et là-bas, mais au lieu d'analyser celle-ci ils la prennent comme point de départ. Selon eux les immigrants n'abandonnent pas leur incorporation politique dans la société de réception quand ils s'engagent dans des pratiques politiques transnationales (Guarnizo et al., 2003). Cependant, loin de faire la preuve d'une incorporation simultanée, les mêmes immigrants qui jouissent des avantages quand il s'agit des relations entretenues avec ceux qui sont restés chez eux se trouvent souvent exclus du système politique là où ils résident. Si les mêmes dollars et les mêmes expériences qui ont des impacts indéniables dans les pays d'émigration n'arrivent pas à améliorer l'incorporation dans le pays d'arrivée, on est en droit de penser que c'est plutôt l'emplacement qui prime sur l'interconnectivité que soulignent, avec tant de ferveur, les transnationalistes. Pour cette raison, des concepts comme ceux de citoyenneté transnationale ou de citoyen transfrontalier ne semblent pas adéquats, surtout pour des personnes qui sont toujours des étrangers, au sens légal.

Mais au-delà de ces critiques, les théoriciens du transnationalisme ont eu le grand mérite de nous rappeler que parler des migrations internationales implique de parler de transnationalisme, ou, plus exactement, d'une variété d'entraides, de flux, de connexions qui vont au-delà d'un territoire en se rattachant à un 
autre. Cependant, la découverte des réseaux traversant les frontières - qu'ils soient quotidien à travers les remises ou communications téléphoniques, ou qu'ils appartiennent à l'action collective menée par les exilés - tient pour acquise la chose même qui est en question. De plus, l'analyse de cet ensemble de liens, d'entraides et d'échanges ne peut que déborder les limites de ce que l'on a appelé le " champ social transnational " qui lie les migrants avec leurs proches résidant dans des lieux lointains. Ce champ-là fait partie d'un champ lui-même plus grand, composé d'acteurs politiques et sociaux, se trouvant à la fois " ici " et " là-bas " et qui répond d'une manière variée et incertaine aux défis et opportunités suscitées par le va-et-vient des personnes, des idées, du capital et des information que suscitent les migrations. Comment caractériser les interactions à travers ce champ complexe englobant non seulement les émigrants et leurs États mais également les États récepteurs et leurs nationaux ?

\section{La citoyenneté à l'étranger}

Du point de vue des États récepteurs, les immigrants sont des étrangers mais pour les États d'origine ces personnes sont des citoyens avec des droits qui les accompagnent partout où ils vont. Les ambassades et les consulats représentent des institutions de souveraineté extraterritoriale où depuis longtemps l'interaction entre l'État et le citoyen se poursuit comme si ce dernier n'avait jamais quitté son lieu d'origine. Puisque tous les États ont des expatriés, ils partagent un intérêt commun à maintenir ce moyen d'accès privilégié. Ces intérêts renforcent les droits accordés aux activités consulaires, ce qui oblige les États récepteurs à permettre aux États émetteurs de réaliser leurs obligations.

La protection des citoyens à l'étranger se trouve au cœur des activités consulaires néanmoins, l'infrastructure consulaire facilite une vaste gamme d'activités à travers lesquelles se rapprochent les uns des autres les nationaux qui se sont implantés dans des territoires étrangers. Quoique le monopole de la force sur les territoires où se trouvent les émigrants échappe aux États émetteurs, ceux-ci continuent à conserver le monopole de l'octroi des documents, ce qui oblige les émigrants - étant donné leur statut étranger sur le sol des états récepteurs - à se dépendre des États d'origine. Le besoin des documents, un passeport, un extrait de naissance ou de décès, une carte d'identité - auquel il faut ajouter des demandes liées aux engagements avec les proches dans le pays d'origine, par exemple, l'expatriation des morts, afin de les enterrer dans la terre natale - est à l'origine de visites régulières au consulat. Des données sur la fréquence de ces visites sont rarement disponibles, mais la source la plus fiable représentée par une enquête auprès deTunisiens, Algériens et Marocains retournés chez eux indique que, pendant leur séjour à l'étranger, les deux tiers d'entre eux ont eu des contacts avec le personnel des consulats, le plus souvent pour des raisons liées aux besoins documentaires. Ainsi, une population qui est sortie du territoire n'est pas perdue de vue : le contact régulier, à travers les fonctions consulaires donne le moyen de suivre les expatriés, ce qui ouvre d'autres opportunités pour les prendre en charge. Le résultat prend différentes formes que ce soit des efforts orientés vers le renforcement des liens culturels, ou des efforts orientés vers la surveillance (Choate, 2009 ; Gamlen, 2008 ; González Gutiérrez, 2006). 
Le fait que les étrangers conservent la citoyenneté d'origine les rapproche donc de leur État d'origine dont ils se sont éloignés, ce qui permet à celui-ci de maintenir les relations avec des populations dispersées au-delà de ses frontières et ainsi de les influencer. Mais le maintien des attaches aux sociétés d'origine n'est qu'une partie d'un double objectif entretenu par les États émetteurs. Ces derniers veulent de surcroît que les émigrants élargissent et stabilisent leur accès aux ressources qui se trouvent dans la société de réception, ce qui entraîne l'acquisition de la citoyenneté de cette même société. Cependant, la poursuite de cet objectif rencontre une entrave sous la forme des sentiments d'appartenance à la société d'origine vivement partagés par les émigrants. Rester citoyen de l'État émetteur fournit peu d'avantages pratiques néanmoins parmi les pays de l'OCDE, presque la moitié des immigrants ne possède pas la citoyenneté du pays d'accueil, un pourcentage encore plus élevé aux États-Unis et en France (OECD, 2011 : 25). La rigidité du statut découle de la nature de l'institution de citoyenneté elle-même, puisque la citoyenneté est à la fois un statut de valeur légale mais aussi le symbole de l'appartenance de l'individu à la nation. Étant donné la pesanteur de cette identification nationale, l'obtention de la citoyenneté du pays de réception peut être perçue comme un acte de trahison (Bloemraad, 2006 ; Gilbertson et Singer, 2003). Ainsi, aux États-Unis, parmi des immigrants d'origine latino-américaine, circule un mythe bien répandu selon lequel les nouveaux citoyens sont obligés de piétiner ou de cracher sur le drapeau de leur pays d'origine pendant la cérémonie de naturalisation (Jones-Correa, 1998 ; Fitzgerald, 2004).

Si un tel mythe - qui n'a aucune relation avec la réalité - reflète néanmoins les conflits émotionnels qui se déroulent dans le for intérieur des émigrants, l'enjeu pour les États émetteurs est celui de savoir comment régler la politique de citoyenneté afin de mieux influencer le comportement des émigrants. S'ils refusent la double citoyenneté, ils renforcent la tendance des émigrants à percevoir l'obtention de la citoyenneté du pays récepteur comme une répudiation du pays d'origine. Leur politique tend donc à encourager la double citoyenneté, ce qui réduit le poids de la loyauté exclusive et permet aux émigrants de se stabiliser dans le pays où ils résident. De fait, les chercheurs ont montré que la promulgation par les États émetteurs des lois de double citoyenneté a la capacité augmente le taux de naturalisation et, parmi les nouveaux naturalisés, les revenus, ce qui est susceptible d'augmenter le flux de remises envoyées par les émigrants au pays d'origine (Mazzolari, 2009).

De telles motivations aident à expliquer la progression de la double citoyenneté, mais ne révèlent qu'une partie de la question. Paradoxalement, la double nationalité est une politique dans laquelle les intérêts des États émetteur et récepteur peuvent converger. Ceux, dans les États récepteurs, qui poursuivent des politiques d'intégration se rendent comptent du fait que l'exigence d'une citoyenneté exclusive empêche l'allégeance des immigrants. De fait, les chercheurs ont montré que l'évolution vers la double citoyenneté dans les États récepteurs exerce un effet semblable à celui qu'on observe dans les États émetteurs, puisque la promulgation des lois permettant la double citoyenneté engendre des taux de naturalisation plus élevés que dans les États récepteurs où la double citoyenneté est refusée (Bloemraad, 2007). Cependant, ceux qui préconisent une politique plus rationnelle ne réussissent pas à convaincre leurs concitoyens. Au lieu de souscrire à une politique mieux adaptée à la situation 
d'une crise d'identité nationale les nationaux des pays récepteurs, convaincus que les loyautés envers la société d'origine représentent une menace, continuent de favoriser une citoyenneté exclusive.

Ces réactions produisent des effets au-delà des aspects identitaires des lois sur la citoyenneté car elles provoquent un durcissement des conditions de naturalisation. Les États européens tendent donc à tolérer la double citoyenneté, tendance qui s'est accrue notablement depuis les années 1990. Néanmoins, les conditions de naturalisation se caractérisent par des exigences de plus en plus grandes; ceux qui tentent d'obtenir la citoyenneté doivent manifester une connaissance beaucoup plus profonde de la langue et de I'histoire du pays récepteur que celle requise il n'y a que dix ans (Howard, 2009). Pour les pays émetteurs, le problème est de n'avoir aucune capacité d'influencer ces tendances néfastes; en conséquence, la portée de la politique poursuivie par les États émetteurs dépend de la politique de naturalisation mise en œuvre par les États récepteurs.

\section{Être membre : la dimension identitaire}

La citoyenneté se réfère aux liens formels rattachant le citoyen à l'État. En revanche, la qualité d'être membre est officieuse, pouvant croître ou décroître d'une façon qui pourrait menacer les intérêts de l'État émetteur. Dans la société d'accueil, l'origine étrangère des immigrants est une source persistante de suspicion surtout si, aux yeux des nationaux, les personnes d'origine étrangère sont toujours celles qui devraient vivre là-bas et non pas ici. D'autre part, les frontières sociales quotidiennes sont faciles à traverser. Aucun immigrant ne veut garder le stigmate de celui qui vient de débarquer; en revanche, les avantages apparaissent rapidement dès qu'on acquiert les outils linguistiques, cognitifs et de communication requis par la société d'accueil. Puisque les migrants appartiennent aux minorités (en termes quantitatifs), ils acquièrent des contacts diversifiés et lorsque la compétence en termes de connaissances et de comportements valorisés par la société de réception augmente, les contacts à travers les frontières ethniques s'élargissent. Ainsi les attaches à la société de réception s'intensifient, tandis que les coûts et les difficultés inhérentes à l'effort de maintenir les contacts avec la société d'origine ne cessent de croître.

Si les sociétés de réception paraissent incapables de se rendre compte de leur puissance assimilatrice, les États émetteurs quant à eux reconnaissent cette réalité. Pour cette raison, ils s'efforcent d'encourager les émigrants qui résident là-bas à ne pas perdre le lien avec l'« ici " d'origine et à maintenir leur sentiment d'appartenance au " nous " national de l'État émetteur (Gamlen, 2008 ; González Gutiérrez, 1999). Parce que cet objectif résonne auprès de certains des émigrants, les deux parties sont enclines à articuler une nouvelle perspective - celle d'être déterritorialisé - dans laquelle la nation se définit comme la communauté qui englobe tous ses citoyens, partout dans le monde où ils se trouvent. En tant que telle, la communauté nationale dépasse les bornes de n'importe quel État et pourrait même dépasser les populations nées dans l'État d'origine pour accorder cet héritage aux descendants des émigrants, nés à l'étranger, et sans aucune expérience sur le sol d'origine. En se plaçant dans cette perspective, les émigrants insistent sur le fait qu'ils sont les mêmes que ceux qui ne se 
sont jamais déplacés de chez eux. Souligner leur allégeance au peuple du pays d'origine sert aussi d'autres buts, notamment pour réclamer des services ou des droits envers les communautés. Paradoxalement, ces revendications sont souvent le produit de l'expérience de l'immigration ; elles reflètent la volonté d'exporter certains des aspects de leurs contacts avec les institutions étrangères - un système politique plus démocratique que celui du pays d'origine, une administration plus rationalisée, prévisible et efficace que celle qu'ils ont connue avant de partir (Goodman et Hiskey, 2008 ; Pérez-Armendáriz et Crow, 2009). Pourtant, les émigrants sont aussi enclins à souligner leurs contributions économiques, reproduisant ainsi le modèle paternaliste ou clientéliste dans leurs interactions avec l'élite gouvernementale ou dans les relations avec les parents qui dépendent des remises qui viennent de l'étranger (Fox, 2005).

Toutefois, les émigrés ne se trouvent pas chez eux, mais plutôt là-bas, ce qui affaiblit leur effort pour revendiquer une allégeance à la communauté nationale du pays où ils ne résident plus. Parmi ceux qui sont restés chez eux, certains perçoivent l'émigration, non comme un départ, mais plutôt comme une désertion et donc un signe de déloyauté. Comme exemple, I'on peut citer l'image défavorable dont souffrent les émigrants dans la culture populaire et dans la politique mexicaines ou encore les qualificatifs utilisés pour décrire les Israéliens qui, en partant pour l'étranger, se voient moins estimés que les Juifs venant de l'étranger et s'installant sur la terre promise. D'autres sources de vulnérabilité proviennent du fait que les migrants, ayant choisi de vivre à l'étranger, sont perçus comme ayant acquis les caractéristiques des étrangers parmi lesquels ils se trouvent. Les migrants peuvent affirmer qu'ils n'ont rien perdu de la culture et des valeurs qu'ils ont connues avant de partir, mais ceux qui restent en contact avec les émigrants sont sensibles aux changements que ces derniers ont subis depuis leur départ vers l'étranger (Fitzgerald, 2009). Puisque l'influence des migrants vient du fait qu'ils se trouvent au-delà du contrôle de l'État d'origine, ils sont perçus par certains des nationaux comme une force étrangère, et même comme un cheval de Troie qui agit pour promouvoir les intérêts de l'État où ils résident (Carpizo et Valadés, 1998). Enfin, puisque les revendications d'allégeance peuvent produire des effets concrets - le déplacement de l'influence vers une population difficile à contrôler, ou des dépenses pour des nationaux qui se trouvent non seulement à l'étranger, mais dans des pays où les coûts sont élevés - les acteurs politiques trouvent des raisons pour insister sur le fait que les nationaux résidant à l'étranger ne jouissent pas de la même appartenance que ceux qui sont restés chez eux.

Les controverses autour du vote à l'étranger mettent en lumière cet aspect ambigu et contesté des revendications des émigrants. Les émigrés exigent quelque chose qu'en tant qu'immigrés ils ne demandent pas : la capacité d'influencer des décisions politiques dont les conséquences ne les affectent pas (Baubock, 2003). De plus, l'argument qu'on avance pour élargir les droits des étrangers dans les pays de réception - l'idée qu'un État démocratique doit inclure tous ceux qui doivent se soumettre à ses lois - fournit la raison pour refuser le droit de vote à ceux qui ne vivent plus dans l'État d'origine et se trouvent en dehors de sa portée (Baubock, 2003 ; Kull, 2008 ; Lopez-Guerra, 2005). Néanmoins, en tant qu'émigrants, les migrants ont généralement réussi à obtenir le droit de vote à l'étranger bien qu'ils aient eu beaucoup moins de succès en tant qu'immigrants cherchant à voter dans les pays où ils résident 
(Baubock, 2003) - ironie qui reflète l'asymétrie de pouvoir entre les migrants et les États émetteurs d'un côté, et ces premiers et les États récepteurs de l'autre. De plus, le fait que des personnes résidant à l'étranger ont le droit de vote a un effet sur la distribution des ressources entre les nationaux vivant " ici " et ceux vivant "là-bas ". Mettre en place la procédure électorale requise pour satisfaire les besoins d'une population dispersée représente un engagement d'une complexité considérable, ce qui explique pourquoi le coût permettant la participation électorale est toujours plus élevé à l'étranger. De surcroît, n'importe quelle opération dans le pays récepteur est plus coûteuse que son équivalent dans le pays d'origine, ce qui la dépense d'impôts payés par ceux qui sont restés chez eux afin de subventionner les votes de nationaux qui généralement ne contribuent pas à ces impôts. Comme la participation électorale à l'étranger est toujours est toujours plus faible que dans le pays d'origine, la valeur des votes migrants tient dans leur qualité symbolique. Cependant, les frais sont loin d'être dérisoires : le Mexique par exemple a dépensé en 2006 plus de 1000 dollars par vote émis à l'étranger (Navarro et Carrillo, 2007).

\section{Participation : la politique des émigrants}

La migration sur le territoire d'un autre pays facilite non seulement l'engagement dans la politique du pays d'origine mais est également source d'une influence inédite. D'abord, l'installation sur le territoire d'un État démocratique permet l'accès aux droits, même si ces derniers sont variables et contestés. À long terme, le matériel et le politique sont complémentaires. La même logique encourageant l'économie familiale transétatique appuie les projets poursuivis par les migrants qui s'engagent dans la politique à longue distance. Dans la mesure où ils réunissent des fonds dans un pays où les salaires sont élevés afin d'aider la mobilisation politique dans les pays où le coût de la vie est moindre, les petites contributions des ouvriers immigrés travaillant dans les situations les plus défavorables fournissent quand même aux exilés les ressources qu'ils requièrent afin d'exercer de l'influence dans le pays d'origine. De plus, même parmi les plus défavorisés des migrants, il y en a toujours qui progressent bien au-delà des positions désavantageuses qu'ils ont initialement occupées. Certains d'entre eux sont prêts à mettre leurs ressources et leurs contacts à disposition des activistes transétatiques. Que les migrants puissent se mobiliser dans un pays plus puissant, jouissant de la capacité d'agir d'une manière qui pourrait aider ou nuire aux régimes des pays d'émigration, ajoute à l'influence que peuvent exercer les expatriés. De plus, le blocage qu'ils auraient subi s'ils étaient restés chez eux se trouve dépassé dans le pays d'accueil où ils arrivent à ériger des alliances avec des acteurs détenant de l'influence dans un pays puissant, ce qui oblige les dirigeants du pays d'émigration à prêter attention à ceux qu'ils ont antérieurement négligés.

Bien entendu, cette possibilité n'a pas la préférence des États émetteurs. Dans la mesure où ces derniers peuvent se servir d'amis bien placés, ils préfèrent plutôt que la politique des émigrants se transforme en politique des immigrants, ce qui permettrait aux immigrants de se convertir en lobbyistes ethniques. L'espoir partagé par tous est d'imiter ce qu'on perçoit être la réussite du lobby juif comme l'a indiqué Jean-Baptiste Aristide il y a trente ans quand en tant que chef de la révolte populaire contre la dictature haïtienne au début des 
années 1990. Il a fait appel aux Haïtiens résidant aux États-Unis pour les encourager à imiter les Juifs américains. Malgré des différences de contexte et d'expérience évidents, l'idée n'est pas seulement partagée par les États émetteurs des pays américains mais se répand bien plus largement. Pour donner un exemple, je citerai le travail de la sociologue Ostergaard-Nielsen (2003: 90), spécialiste de la migration turque qui rapporte qu'un membre de l'Assemblée turque lui a dit que " tout le monde veut un lobby juif ".

Mais le mariage entre politique des émigrants et politique des immigrants n'est pas si facile à réaliser. Puisque les émigrants se sont soustraits au contrôle de l'État émetteur, ils peuvent poursuivre la politique en tant qu'immigrants afin de mettre en place une politique opposée à celle préconisée par l'élite dans le pays d'origine. L'exemple des Cubains illustre ce modèle. Dès qu'ils touchent le sol américain, les Cubains bénéficient de la résidence permanente et demandent le plus rapidement possible la citoyenneté. Une fois celle-ci obtenue ils exercent massivement leur droit de vote. De plus ils se concentrent dans les États de Floride et du New Jersey où le résultat des élections présidentielles est souvent décisif. Enfin, ils votent en bloc ce qui les rend attractifs aux yeux des candidats. En conséquence les partis démocrate et républicain s'efforcent de soutenir le programme mis en avant par les exilés qui vise à accroître la pression subie par le régime cubain (Eckstein, 2009). Évidemment, le cas cubain est loin d'être le seul. Une migration aussi apolitique que la migration mexicaine s'est révélée capable d'une mobilisation en tant que citoyens américains afin de faire pression sur le gouvernement mexicain.

La politique des émigrants peut donc facilement échapper au contrôle des États d'origine. De plus, l'équilibre entre les loyautés ressenties envers le pays d'émigration et les attentes du peuple auquel on vient d'adhérer est un dilemme qui n'est pas facile à résoudre. Les émigrants veulent maintenir les liens qui les rattachent aux pays d'origine sans pour autant rappeler aux nationaux de l'État de réception que ceux-ci ont accueilli des personnes d'origine étrangère ayant maintenu des attaches aux pays étrangers. Dans le meilleur des cas, les publics des pays récepteurs sont enclins à tolérer et souvent même à accepter les loyautés rattachant les immigrants à leurs pays d'origine, mais ils restent jaloux prêts à percevoir dans les manifestations d'attachement au pays d'origine comme un abus de leur générosité (Harrington, 1980). Au début des années 1990, bien avant que les événements du 11 septembre aient poussé le public américain à se méfier des liens des immigrants à l'égard de leurs pays d'origine, les données d'enquêtes, venant d'échantillons nationaux représentatifs, ont démontré que selon le public, les Américains-juifs, -noirs, -asiatiques ou -hispaniques manifestent des loyautés divisées et par conséquent ne font pas preuve du même niveau de patriotisme que les Américains sans "trait d'union " (Smith, 1991). De plus, une minorité importante déjà inquiète de l'arrivée de tant d'étrangers pense que les revendications de double allégeance vont beaucoup trop loin. Même si tous ne partagent pas la vue extrême du journaliste Geyer (1996) qui dans son livre The Death of American Citizenship, a comparé la double nationalité à la bigamie. Néanmoins, il ne serait pas surprenant d'apprendre que ceux qui conçoivent la nation comme une famille à large échelle se fâchent en voyant des défilés dans lesquels les immigrants brandissent les drapeaux des États d'émigration (Huntington, 2004). 


\section{Conclusion}

En tout temps et en tous lieux, les migrations entraînent l'extension des réseaux de populations, d'informations et des biens au-delà du territoire de l'État. En partant d'un pays pour un autre, les migrants se déplacent d'une société au territoire d'un autre État ce qui produit une convergence des sociétés. La manière de comprendre ce déplacement dépend du lieu depuis lequel on l'observe. Du point de vue des États récepteurs et de leurs peuples, la migration entraîne l'arrivée, ici, de personnes étrangères qui auraient dû rester là-bas dans les terres étrangères. Le point de vue à l'autre bout de la chaîne n'est pas tellement différent, puisque la totalité du " nous " national ne se trouve plus " ici " chez nous mais plutôt " là-bas " dans les terres étrangères.

Les migrants, en revanche, se trouvent entre les deux rives, situation qui leur fournit des ressources qui comptent dans les relations avec les parents, les communautés et l'État du lieu d'origine. Cependant, bien que dans les relations avec ceux qui restent là-bas les migrants tirent des avantages de leur mouvement d'un pays à l'autre, la condition d'étranger - au sens social comme au sens juridique - les laisse vulnérables. Ces deux situations déclenchent l'action des États émetteurs qui essaient tout à la fois de protéger et de contrôler ceux qui ne se trouvent plus sur le territoire d'origine. Mais le fait que tout se déroule sur le territoire d'un autre État complique le jeu. L'État émetteur peut suivre ces citoyens sur le territoire d'un autre État mais en se déplaçant il perd sa puissance et ne conserve que de l'influence. Bien que les migrants revendiquent leur appartenance au " nous " de la société d'origine, ceux qui sont restés chez eux n'ont pas la même opinion car ils pensent que les migrants ne sont plus au cœur comme "nous " mais plutôt comme " eux " parmi lesquels ils vivent. De fait, ils ne se trompent pas totalement puisqu'au fur et à mesure que le séjour des émigrants à l'étranger se poursuit ces derniers se différencient de ceux qui sont restés chez eux, ce qui donne un caractère étranger aux revendications dirigées vers la communauté d'origine. Pour les nationaux de la société de réception, les immigrants ne sont pas les bienvenus, non seulement parce qu'ils font partie $d^{\prime}$ " eux ", et pas de " nous " mais aussi parce qu'ils viennent de là-bas. Cette double réaction, contre les étrangers et les pays étrangers d'où ils viennent, augmente la vulnérabilité des migrants et limite la capacité de l'État émetteur à établir sa présence sur un sol étranger. Pour les immigrants qui sont aussi des émigrants, il en résulte un dilemme puisqu'ils veulent être à la fois " eux " et " nous ", ici et là-bas, dans une quête qui a peu de chance d'être couronnée de succès. En revanche, pour les chercheurs ce dilemme est une bonne nouvelle parce que la complexité ajoute toujours de l'intérêt à la recherche. Mais pour apprécier ce dilemme et pour en tirer de potentiels bénéfices il faut dépasser la division entre ceux qui focalisent leur attention sur l'immigration et ceux qui se concentrent sur l'émigration. II faut plutôt imiter ces immigrants qui sont également des émigrants, en cherchant à les suivre d'une rive à l'autre dans le but de comprendre ce qui lie mais aussi ce qui sépare ici et là-bas. 


\section{Références bibliographiques}

Basch Linda, Glick Schiller Nina and Szanton Blanc Cristina (1994) Nations Unbound:Transnational Projects, Postcolonial Predicaments, and Deterritorialized Nation States, New York, Gordon and Breach, 356 p.

Bauböck Rainer (2005) Expansive Citizenship. Voting beyond Territory and Membership, Political Science \& Politics, 38, pp. 683-687.

Bauböck Rainer (2003) Toward a Political Theory of Migrant Transnationalism, International Migration Review, 37 (3), pp. 700-723.

Bloemraad Irene (2007) Much Ado about Nothing? The Contours of Dual Citizenship in the United States and Canada, in Thomas Faist and Peter Kivisto, Dual Citizenship in Global Perspective, New York, Palgrave, pp. 159-188.

Bloemraad Irene (2006) Becoming a Citizen: Incorporating Immigrants and Refugees in the United States and Canada, Berkeley, UC Press, 382 p.

Carpizo Jorge y Valadés Diego (1998) El Voto de los Mexicanos en el Extranjero, México City, Universidad Nacional Autónoma de México, 140 p.

Choate Mark (2009) Emigrant Nation: The Making of Italy Abroad, Cambridge, Harvard University Press, $340 \mathrm{p}$.

Dufoix Stéphane (2002) Politiques d'exil, Hongrois, Polonais, Tchécoslovaques en France après 1945, Paris, Presses Universitaires de France, 328 p.

Dufoix Stéphane, Guerassimoff Carine et Tinguy Anne de (Éds.) (2010) Loin des yeux, près du coeur: les États et leurs expatriés, Paris, Presses de Sciences Po, $341 \mathrm{p}$.

Eckstein Susan (2009) The Immigrant Divide: How Cuban Americans Changed the U.S. and their Homeland, New York, Routledge, 312 p.

Fitzgerald David (2009) A Nation of Emigrants. How Mexico manages its migration, Berkeley, University of California Press, $264 \mathrm{p}$.

Fitzgerald David (2004) Beyond "transnationalism": Mexican hometown politics at an American labour union, Ethnic and Racial Studies, 27 (2), pp. 228-247.

Fox Jonathan (2005) Unpacking Transnational Citizenship, Annual Review of Political Science, 8, pp. 171-201.

Gamlen Alan (2008) The emigration state and the modern geographical imagination, Political Geography, 27, pp. 840-856.

Geyer Georgie Anne (1996) Americans No more. The Death of American Citizenship, New York, Atlantic Monthly Press, 352 p.

Gilbertson Greta and Singer Audrey (2003) The emergence of protective citizenship in the USA: naturalization among Dominican immigrants in the post-1996 welfare reform era, Ethnic and Racial Studies, 26 (1), pp. 25-51.

González Gutiérrez Carlos (2006) Introducción: El Papel de los Gobiernos, in Carlos González Gutiérrez Ed., Relaciones Estado-diáspora: La perspectiva de América Latina y el Caribe, México, DF, Porrua, pp. 13-42.

González Gutiérrez Carlos (1999) Fostering Identities: Mexico's Relations with Its Diaspora, The Journal of American History, 86 (2), pp. 545-567. 
Goodman Gary and Hiskey John (2008) Exit without Leaving: Political Disengagement in High Migration Municipalities in Mexico, Comparative Politics, 40, pp. 169-188.

Green Nancy et Weil Françoịs (2006) Citoyenneté et émigration. Les politiques du départ, Paris, Éditions de l'École des Hautes Études en Sciences Sociales, 274 p.

Guarnizo Luis, Portes Alejandro and Haller William J. (2003) Assimilation and Transnationalism: Determinants of Transnational Political Action among Contemporary Migrants, American Journal of Sociology, 108 (6), pp. 1211-1248.

Harrington Mona (1980) Loyalties: Dual and Divided, in Stephan Thernstrom Ed., Harvard Encyclopedia of American Ethnic Groups, Cambridge, MA, Harvard University Press, pp. 676-686.

Howard Marc (2009) The Politics of Citizenship in Europe, New York, Cambridge University Press, $258 \mathrm{p}$.

Huntington Samuel (2004) Who Are We: The Challenges to America's National Identity, New York, Simon \& Schuster, 448 p.

Iskander Natasha (2010) Creative State: Forty Years of Migration and Development Policy in Morocco and Mexico, Ithaca, NY, Cornell University Press, 392 p.

Itzigsohn Jose (2000) Immigration and the Boundaries of Citizenship, International Migration Review, 34 (4), pp. 1126-1154.

Jones-Correa Michael (1998) Between two nations: The political predicament of Latinos in New York City, Ithaca, NY, Cornell University Press, 272 p.

Kull Christian A. (2008) Who should vote where? Geography and Fairness in Migrant Voting Rights, Geographical Research, 46 (4), pp. 459-465.

Levitt Peggy and Jaworsky B. Nadya (2007)Transnational Migration Studies: Past Developments and FutureTrends, Annual Review of Sociology, 33, pp. 129-155.

Lopez-Guerra Claudio (2005) Should Expatriates Vote?, Journal of Political Philosophy, 13 (2), pp. 216-234.

Mazzolari Francesca (2009) Dual Citizenship rights: do they make more and richer citizens, Demography, 46 (1), pp. 169-191.

Navarro Fierro Carlos and Carillo Manuel (2007) Mexico: Safeguarding the Integrity of the Electoral Process, in IDEA, Voting from Abroad: The International IDEA Handbook, Sweden, International IDEA, pp. 189-192.

OECD (Organization for Economic Cooperation and Development) (2011) Naturalisation: An Instrument for the Better Integration of Immigrants?, Paris, OECD, $356 \mathrm{p}$.

Ostergaard-Nielsen Eva (Ed.) (2003) International Migration and Sending Countries: Perceptions, Policies, and Transnational Relations, London, Palgrave, $246 \mathrm{p}$.

Pérez-Armendáriz Clarissa and Crow David (2009) Do Migrants Remit Democracy? International Migration and Political Beliefs and Behavior in Mexico, Comparative Political Studies, 42 (9), pp. 1-28.

Portes Alejandro and Rumbaut Rubén (2006) Immigrant America: A Portrait (3rd ed.), Berkeley, University of California Press, 460 p. 
Saunier Pierre-Yves (2009) Transnationalism, in Arika Iriye and Pierre-Yves Saunier Eds., The Palgrave Dictionary of Transnational History, New York, Palgrave, pp. 1047-1055.

Shain Yossi (1989) The Frontier of Loyalty, Middletown, CT, Wesleyan University Press, $214 \mathrm{p}$.

Smith Michael Peter and Guarnizo Luis Eduardo (1998) Transnationalism from Below. Comparative Urban and Community Research, New Brunswick, N.J., Transaction Publishers, $316 \mathrm{p}$.

Smith Tom W. (1991) What Do Americans Think About Jews?, New York, American Jewish Committee, 70 p.

Waldinger Roger (2015) The Cross-border Connection: Immigrants, Emigrants, and their Homelands, Cambridge, MA, Harvard University Press, 240 p.

Waldinger Roger (2011) Transnationalism and the Sociology of Immigrants' Cross-Border Connections, Sociopedia.isa, pp. 1-13.

Waldinger Roger (2006) "Transnationalisme " des immigrants et présence du passé, Revue Européenne des Migrations Internationales, 22 (2), pp. 23-42.

Waldinger Roger and Fitzgerald David (2004) Transnationalism in Question, American Journal of Sociology, 109 (5), pp. 1177-1195. 


\section{Roger Waldinger}

\section{La politique au-delà des frontières : la sociologie politique de l'émigration}

Cet article propose un cadre d'analyse de la sociologie politique de l'émigration. II met l'accent sur les dualités logées au cœur du phénomène migratoire : les immigrants sont aussi des émigrants, les étrangers au sens juridique (aliens) sont aussi des citoyens, les étrangers au sens social (foreigners) sont aussi des nationaux, les exclus des sociétés de réception sont aussi les membres des sociétés d'origine. De la société d'origine mais n'étant plus dans cette société, les migrants sont des membres dont les connexions transfrontalières et les besoins d'adaptation poussent l'État émetteur à s'élargir au-delà des frontières, mais le fait de résider à l'étranger affaiblit leurs revendications d'appartenance. Dans la société de réception mais n'étant pas de cette société, ils ont accès à des ressources économiques et politiques qui leur permettent d'exercer de l'influence dans la société d'origine, mais en tant qu'étrangers leurs droits sont limités et leur acceptation incertaine, vulnérabilité qui peut s'aggraver lorsqu'un engagement continué à l'égard du pays d'origine suscite la suspicion des nationaux de l'État récepteur. Cette variété de conditions déclenche des interventions des États émetteurs qui cherchent à protéger et à influencer leurs nationaux résidant à l'étranger mais également à répondre aux revendications de ces citoyens à l'extérieur du pays et à les canaliser afin de mieux les récupérer. Cependant, l'extension au territoire d'un pays étranger empêche l'exercice du pouvoir et ne permet que l'exercice d'une influence. Et dans ce cas des interventions même limitées courent le risque d'enflammer les passions des nationaux déjà inquiets par la présence d'une population étrangère parmi eux.

\section{Politics beyond Borders:}

\section{The Political Sociology of Emigration}

This paper seeks to develop a framework for analyzing the political sociology of emigration. It emphasizes the dualities at the heart of the migration phenomenon: immigrants are also emigrants, aliens are also citizens, foreigners are also nationals, non-members are also members. At once of the sending state, but not in it, the migrants are members whose everyday cross-border connections and ongoing needs draw the sending state across the borders; residing abroad, however, their claims to belonging are undermined by their presence on foreign soil. At once in the receiving state but not of it, the migrants can access the economic and political resources available in their new home, using them to gain leverage in the home left behind; yet as outsiders, their rights are circumscribed and their acceptance is uncertain, vulnerabilities that can be aggravated if continuing homeland involvement triggers the suspicion of receiving state nationals. Both conditions activate interventions by home states seeking to influence and protect nationals abroad. While extension to the territory of another state keeps options inherently limited, even limited engagements can inflame the passions of receiving state nationals, already anxious about the foreigners in their midst. 


\section{Política más allá de las fronteras: la sociología política de la emigración}

Este artículo pretende desarrollar un marco de análisis de la sociología de la emigración. Para ello se centra en las distintas dualidades intrínsecas al fenómeno migratorio: los inmigrantes son a la vez emigrantes, los extranjeros son al mismo tiempo ciudadanos, los extranjeros también son nacionales, los "no-miembros" son a la vez miembros. Provenientes de un Estado en el que ya no residen; los migrantes mantienen con su lugar de origen una conexión diaria y transfronteriza y sus necesidades de adaptación hacen que el Estado de origen se extienda más allá de sus fronteras. No obstante, el hecho de residir en el extranjero hace que sus reivindicaciones se vean debilitadas. Residiendo en el extranjero, aunque sin formar parte del país de acogida, los migrantes pueden acceder a los recursos económicos y políticos disponibles en su nuevo hogar, revirtiendo estos en el hogar que dejaron atrás. Sin embargo, el hecho de ser extranjeros implica al mismo tiempo que sus derechos son limitados y su aceptación por parte de la sociedad de acogida es incierta; una vulnerabilidad que puede agravarse si se produce un cierto compromiso con el país de origen y que este suscita la sospecha de los nacionales del Estado de acogida. Ambas condiciones llevan a los Estados de origen a elaborar políticas dedicadas a sus nacionales y su protección en el extranjero. Si bien es cierto que la extensión de un Estado al territorio de otro es una opción en sí misma muy limitada, esos pequeños compromisos pueden agitar a los nacionales del estado de acogida, ya preocupados por la presencia de extranjeros entre ellos. 